\title{
A Complexidade e a Estabilidade da Estrutura do Conhecimento Tecnológico na Evolução dos Micro-paradigmas
}

\author{
Vanessa de Lima Avanci ${ }^{1}$ \\ Ana Urraca Ruiz ${ }^{2}$
}

\begin{abstract}
Resumo
Nas últimas décadas houve uma forte expansão da quantidade de patentes com o aumento também da quantidade de relações novas entre campos tecnológicos diferentes, ainda assim vários estudos apontam para a estabilidade da estrutura do conhecimento tecnológico. Ao emergir, os paradigmas tecnológicos criam oportunidades de pesquisa, de avanço do conhecimento tecnológico e levam a uma maior integração entre campos tecnológicos distintos. A estrutura do conhecimento tecnológico se torna mais complexa através da integração dos seus distintos componentes e cria limites às direções de pesquisa e de acumulação. Os objetivos deste trabalho são comparar a estrutura de um mapa de patentes global em períodos distintos no tempo, identificar os seus principais componentes e averiguar se a estabilidade é uma característica intrínseca a essa estrutura. A análise das redes formadas pelas patentes em 1988, 1998 e 2008 e dos agrupamentos dos campos tecnológicos mostrou que a estrutura do conhecimento tecnológico geral não se manteve estável ao longo do tempo. Os resultados também mostraram que um núcleo constituído por relações entre campos tecnológicos se tornou relativamente mais importante ao longo do tempo.
\end{abstract}

Palavras-chave: Complexidade Tecnológica; Estrutura de conhecimento; Paradigmas e Trajetórias tecnológicas; Distância Tecnológica; Patentes.

\begin{abstract}
In the last decades there has been a strong expansion of the number of patents with an increase in the number of new relations between different technological fields, yet several studies point to the stability of the technological knowledge structure. When emerging, technological paradigms create opportunities for research, advancement of technological knowledge and lead to greater integration between different technological fields. The structure of technological knowledge becomes more complex through the integration of its distinct components and creates limits to the directions of research and accumulation. The objectives of this work are to compare the structure of a global patent map at different time periods, to identify its main components and to investigate whether stability is an intrinsic characteristic of this structure. The analysis of networks formed by patents in 1988, 1998 and 2008 and of the clusters of technological fields showed that the structure of general technological knowledge has not remained stable over time. The results also showed that a core composed of relations between technological fields became relatively more important over time.
\end{abstract}

Keywords: Technological Complexity; Knowledge structure; Technological Paradigms and Trajectories; Technological Distance; Patents.

ÁREA ABEIN: 5.9 Indicadores de Ciência, Tecnologia, Inovação

CLASSIFICAÇÃO JEL: C81; O31

\footnotetext{
${ }^{1}$ Doutoranda no Programa de Pós-Graduação em Economia da Universidade Federal Fluminense (PPGE-UFF).

${ }^{2}$ Professora Associada do Departamento de Economia da Universidade Federal Fluminense (UFF).
} 


\section{Introdução}

A tecnologia é um conjunto de peças de conhecimento de caráter prático e teórico, que envolvem métodos e procedimentos e que se encontram incorporadas em artefatos (Dosi, 1984). Um conjunto de tecnologias representa, desta forma, uma base de conhecimento formada pelo conjunto de peças interrelacionadas de conhecimento científico e tecnológico acumuladas mediante o aprendizado e a experiência. As peças de conhecimento que compõem a base são de diversas naturezas e atendem a diferentes 'campos tecnológicos' de acordo com sua função ou aplicação [por exemplo: química, eletrônica, etc.]. Quando elas se combinam, formam estruturas de conhecimento com relações altamente complexas.

Os ciclos de inovações marcados pelo surgimento de novos paradigmas tecno-econômicos tendem a modificar as estruturas de conhecimento de distintas formas. As inovações de caráter incremental normalmente são novas combinações de campos tecnológicos previamente relacionados. Já as inovações radicais alteram a estrutura do conhecimento ao incluir novos campos, estabelecer novas composições ou ambos, isto é, associar campos tecnológicos que não existiam ou não se relacionavam previamente. Por tanto, a evolução da estrutura do conhecimento consiste na mudança das relações entre os campos tecnológicos e supõe um passo inicial para o estudo das trajetórias seguidas pelo progresso técnico. Paralelamente, quando um novo paradigma emerge, a direção e o ritmo de progresso técnico são incertos. Mas à medida que os padrões tecnológicos vão se estabelecendo, as incertezas se reduzem e as diversas direções do progresso técnico vão se assentando. Assim, uma trajetória tecnológica representa um cluster de direções tecnológicas possíveis, cujos limites externos são definidos pelo paradigma (Dosi, 1984). Por isso, as trajetórias ordenam e limitam as direções de avanço tecnológico, mas não eliminam a variedade de possibilidades que surge dos processos de busca. As trajetórias podem seguir múltiplas direções sobre uma diversidade tecnológica e esses diversos caminhos podem evoluir independentemente ou co-evoluir uns com os outros. Em sistemas tecnológicos complexos, a co-evolução de diversos campos tecnológicos é necessária. Se alguns campos de conhecimento não evoluírem no mesmo ritmo que os seus complementares, geram-se situações de lock-in freando o desenvolvimento da trajetória (Sahal, 1985).

Entre as fases de surgimento e amadurecimento de um paradigma tecno-econômico existem forças diferentes influenciando a estrutura de conhecimento. $O$ surgimento de um paradigma e os desdobramentos de novas trajetórias representam oportunidades tecnológicas para novas combinações de peças de conhecimento e, portanto, mudanças na estrutura. À medida que os paradigmas amadurecem, a base de conhecimento tende a ficar mais complexa, isto é, as trajetórias tecnológicas vinculadas a micro paradigmas similares se cruzam dando lugar a crescentes graus de complexidade que se revelam em produtos e artefatos também mais complexos. Quando estabelecidos, os paradigmas também criam limites para o desenvolvimento de novas trajetórias. Por estarem vinculados a padrões e métodos específicos de resolução de problemas, tendem a reforçar as relações que já existem entre os campos tecnológicos, tornando assim a base de conhecimento estável, localmente específica (Granstrand et al., 1997; Patel e Pavitt, 1997) e com um elevado grau de irreversibilidade ou path-dependence (Breschi et al., 2003; Pavitt, 1984; Malerba e Orsenigo, 1996).

Uma maior complexidade da base de conhecimento tecnológico ao longo do tempo significa uma maior integração entre campos tecnológicos distintos. As fases de desenvolvimento, produção e uso dos novos artefatos envolvem o uso de tecnologias que tem origem em campos técnicos diferentes; e algumas tecnologias são aplicadas a vários campos técnicos distintos [permeabilidade]. As tecnologias permeáveis, quando combinadas em diversas aplicações tecnológicas, aumentam a necessidade de integração do conhecimento de distintos campos técnicos, gerando conhecimento local e específico [convergência] (Grandstrand e Slojander, 1990). A evolução dos paradigmas tecnológicos nas últimas décadas gerou uma forte convergência tecnológica entre industrias, levando a um crescimento da conexão entre campos tecnológicos. Porém, os instrumentos metodológicos relativos à análise de redes não registram mudanças significativas na relação entre as tecnologias ao longo do tempo (Joo e Kim, 2010). 
Em termos aplicados, o seguimento da evolução de uma estrutura de conhecimento tecno-científico permite identificar a seletividade e persistência [estabilidade] das trajetórias tecnológicas a partir das inter-relações entre as peças de conhecimento que a compõem. A seletividade está associada à convergência das trajetórias ao longo do tempo, isto é, das diversas tecnologias que emergem, só algumas evoluem seguindo trajetórias específicas. A persistência das trajetórias se refere à cumulatividade, ou seja, ao como a inovação ocorre sobre conhecimento existente e como as trajetórias passadas contribuem para a continuidade e também para as novas direções das inovações. A incerteza inerente à busca de novas tecnologias sob influência de fatores econômicos e sociais pode levar a mudanças na direção da trajetória tecnológica. Como consequência, o grau de persistência é variável no tempo dependendo da combinação de períodos em que se exploram novas trajetórias ou em que se acumula conhecimento dentro dos limites de trajetórias dominantes (Verspagen, 2007). O amadurecimento de alguns dos paradigmas tecno-econômicos que surgiram nos anos oitenta estabilizou as trajetórias e as relações entre campos tecnológicos existentes. A estabilidade se caracteriza pela permanência ao longo do tempo de tecnologias núcleo em torno das quais se estabeleceram numerosas e fortes relações entre campos tecnológicos; enquanto que a complexidade se manifesta na integração cada vez maior dos campos técnicos que formam a estrutura do conhecimento. O surgimento de novas trajetórias tecnológicas e a superação de situações de lock-in criam oportunidades de novas combinações, o que deve ser observado no surgimento de novas relações entre os campos tecnológicos e deve causar alterações na estrutura da base de conhecimento tecnológico.

Neste sentido, o objetivo geral deste trabalho é analisar a evolução da estrutura do conhecimento ao longo de três décadas (78-1988, 89-1998 e 99-2008) e identificar como a evolução dos novos paradigmas tecnológicos alterou a estrutura em termos de complexidade e estabilidade das inter-relações entre peças de conhecimento. Para isto, o trabalho se divide em três partes além desta introdução. Primeiro, o artigo estabelece as relações dos paradigmas tecnológicos e trajetórias tecnológicas com a evolução da estrutura do conhecimento tecnológico. Em seguida, o artigo apresenta uma revisão das formas de representação da estrutura do conhecimento tecnológico e suas principais contribuições para a compreensão das relações entre campos tecnológicos. A última parte propõe uma nova forma de analisar a estrutura de conhecimento tecnológico que associa os aspectos de estabilidade e mudança ao amadurecimento dos paradigmas e ao surgimento de novas trajetórias tecnológicas, respectivamente.

\section{Representação da estrutura do conhecimento tecnológico e sua evolução a partir de dados de patentes}

As patentes são comumente usadas para analisar as atividades de invenção e inovação, pois revelam resultados de esforços formais e informais em P\&D. Além disso, as bases de dados de patentes são consideradas boas fontes de atividade tecnológica, por oferecerem informações detalhadas por setor para um grande número de países para longos períodos de tempo. Uma patente representa um avanço no estado da arte de uma tecnologia e trata apenas do conhecimento codificado que pode ou não ser incorporado em artefatos ${ }^{3}$. Neste sentido, as patentes dão apenas uma ideia parcial da complexidade do conhecimento. Além disso, nem todos os esforços de mudança tecnológica resultam em patentes, por isso, tecnologias não patenteáveis ou escassamente protegidas sob patentes não são consideradas nas estruturas de conhecimento construídas a partir de informações de patentes ${ }^{4}$. As estatísticas de patentes também estão sujeitas a um viés de distribuição setorial, dado que sua eficácia como forma de apropriação varia entre indústrias e entre tecnologias, o que pode subestimar algumas relações entre campos tecnológicos.

\footnotetext{
${ }^{3}$ As capacitações envolvem indicadores de conhecimento incorporado e não incorporado, conhecimento tácito e codificado e geração e difusão de conhecimento, mesmo existindo complementaridades entre essas categorias (Archibugi e Coco, 2005).

${ }^{4}$ As competências tecnológicas podem não resultar em patentes por não se basearem em tecnologias patenteáveis ou por decisões estratégicas das firmas.
} 
Cada patente tem atribuído um código que classifica o conhecimento associado a um determinado campo de conhecimento técnico (International Patent Classification. IPC) para diferentes níveis de agregação. A classificação IPC estabelece categorias ordenadas hierarquicamente que representam processos, produtos, aparatos ou materiais agrupados em temas que tratam da natureza, função, forma de uso ou aplicação da invenção na indústria. Como a base de conhecimento que é usada nas indústrias é pouco flexível, a classificação IPC tende a ser relativamente estável. Quando a patente representa uma tecnologia que combina peças de conhecimento de diferentes naturezas, lhe são atribuídos mais de um código IPC, em cujo caso diz-se que há coocorrência.

Existem duas formas de usar as bases de dados de patentes para construir a estrutura de relações entre os campos de conhecimento: mediante citações de patentes e por coocorrência de campos tecnológicos. As citações de patentes relacionam tecnologias [e campos tecnológicos] ao longo do processo de acumulação de conhecimento e permitem observar a direção e o ritmo da mudança tecnológica a partir da recombinação de peças de conhecimento ao longo do tempo, assim como a emergência de descontinuidades em tecnologias específicas (Verspagen, 2007; Mina et al., 2007; Fontana et al., 2009). A coocorrência indica mais diretamente o grau de complexidade tecnológica do que as citações. Como mais de um código de classificação é atribuído a uma patente quando a inovação se refere a aspectos técnicos distintos, esses códigos tendem a ser atribuídos juntamente quando os campos tecnológicos representados pelos códigos estão relacionados (Joo e Kim, 2010). A análise de coocorrência é utilizada para medir o grau de relação entre dois campos tecnológicos, à semelhança de trabalhos que medem a relação entre indústrias (Hidalgo et al., 2007; Teece et al., 1994; Bryce e Winter, 2009) e entre tópicos de pesquisa (Heimeriks e Boschma, 2012).

O mapeamento de trajetórias tecnológicas é uma representação simbólica dos campos tecnológicos contidos numa patente e suas interconexões. Os mapas realizados por Hinze et al. (1997) identificaram que existiam semelhanças entre países (Estados Unidos, Japão e Alemanha) e distintos períodos (19821985, 1986-1989 e 1990-1993) concluindo que existe certo grau de estabilidade na distribuição entre campos tecnológicos. Isso permitiria que a estrutura da base de conhecimento tecnológico fosse comparável no tempo e para diferentes níveis de análise, desde a firma até o país. O autor utilizou o conceito de distância tecnológica como o grau em que as peças de conhecimento tecnológico se combinam para gerar conjuntos específicos de patentes, ou seja, quanto maior a proximidade entre tecnologias, maior é a oportunidade de inovação (Breschi et al., 2003). A distância entre campos tecnológicos (similaridade ou proximidade) determina o potencial de inovação e a variedade de tecnologias disponíveis (Nooteboom, 2007; Levinthal e March, 1993; Saviotti e Frenken, 2008 in Schoen, 2012). A posição relativa dos campos tecnológicos no mapa pode indicar o potencial de recombinação do conhecimento (Fleming, 2001; Nakamura et al., 2015), assim como direções e trajetórias de diversificação tecnológica (Boschma, Balland e Kogler, 2015; Breschi, Lissoni e Malerba, 2003; Rigby, 2013), e tecnologias emergentes (Benson e Magee, 2013, 2015; Kajikawa et al., 2008; Ogawa \& Kajikawa, 2015). A proximidade entre as tecnologias medida através de citações de patentes não se estabelece necessariamente por pertencerem ao mesmo corpo de conhecimento, podendo refletir outras características de aplicação das tecnologias por similaridade, complementaridade ou coocorrência (Kay et al., 2014).

A medida de distância tecnológica aplicada a uma base acumulada de conhecimento tecnológico [peças de conhecimento inter-relacionadas] permite não só observar o desenvolvimento da tecnologia em relação às suas trajetórias passadas, mas também as possibilidades futuras. As patentes que referenciam campos tecnológicos próximos sugerem oportunidades para inovação incremental, enquanto patentes que referenciam campos tecnológicos distantes ou combinações mais incomuns tem um potencial maior para inovações radicais (Olsson, 2004 in Kay et al., 2014; Aharonsona e Schilling, 2016). As inovações microradicais estão associadas ao refinamento progressivo das capacidades estabelecidas pelos paradigmas e, portanto, constroem a base de conhecimento tecnológico sobre campos tecnológicos que se relacionavam previamente. Um exemplo recente é o caso das tecnologias ambientais. Com o crescimento da quantidade de informações que apareceram nas patentes se referindo a tecnologias ambientais, o Escritório de 
Patentes Europeu (EPO) desenvolveu um esquema de classificação próprio que permitiu mapear essas tecnologias (Veefkind et al., 2012). As tecnologias ambientais surgiram primeiramente como uma seleção de campos tecnológicos que existiam previamente. No entanto, à medida que o micro-paradigma se desenvolveu, diferentes trajetórias tecnológicas [tecnologias associadas a esses campos tecnológicos] foram emergindo (Mitigação de GHG; Energias renováveis; Emissão de gases; Biocombustíveis; etc..).

\section{A Estabilidade da estrutura do conhecimento tecnológico associada aos paradigmas tecnológicos}

A base de patentes do Escritório de Patentes Europeu (EPO) para o período de 1978-2008 conta com um total de 2,474,208 patentes. Como cada patente pode ter associado mais de um campo técnico, a base de conhecimento contou com um total de 4,758,624 registros para um nível de agregação de 3 dígitos (121 campos tecnológicos).

O mapa geral representa uma rede onde os campos técnicos são os nós e as coocorrências entre campos são os vínculos (links). O grau de um nó é determinado pelo número de campos tecnológicos conectados a ele por ao menos uma aresta e o grau médio da rede é a média aritmética do grau de todos os nós. A densidade se define pelo quociente entre o número de campos técnicos conectados sobre o número de campos técnicos totais da rede. $O$ valor da densidade da rede varia entre 0 e 1 , sendo que 1 representa uma rede em que todos os componentes estão conectados e 0 uma rede de componentes não conectados. Ao longo das três últimas décadas houve crescimento do grau médio da rede de $84 \mathrm{em} \mathrm{1988,} \mathrm{para} 97 \mathrm{em}$ 1998 e para 105 em 2008 (Tabela 1). Ao mesmo tempo, a rede foi se adensando cada vez mais. A quantidade total de nós da rede se manteve em 121 em todos os períodos, mas surgiram novos links entre campos tecnológicos que não se relacionavam previamente. $\mathrm{O}$ valor da densidade da rede revela que os campos de conhecimento tecnológico estão muito conectados nesta estrutura e que a integração aumentou muito, particularmente entre 1988 e 1998.

Tabela 1. Grau médio e densidade da rede

\begin{tabular}{lccc}
\hline & $\mathbf{1 9 8 8}$ & $\mathbf{1 9 9 8}$ & $\mathbf{2 0 0 8}$ \\
\cline { 2 - 4 } Grau médio & 84 & 98 & 105 \\
Densidade & 0,698 & 0,816 & 0,874 \\
Total de nós & 121 & 121 & 121 \\
\hline
\end{tabular}

Fonte: Elaboração própria.

O adensamento da rede significa que o surgimento de novas relações entre campos tecnológicos acompanhou mais do que proporcionalmente o crescimento mundial da atividade patenteadora. Apesar da diferente propensão a patentear entre tecnologias e, em consequência, da diferente probabilidade de frequência entre campos tecnológicos, estes estão se tornando cada vez mais inter-relacionados, o que já revela o aumento da complexidade da base de conhecimento tecnológico. A integração da estrutura do conhecimento tecnológico, porém, não ocorreu de forma equilibrada. Algumas relações entre campos tecnológicos ocorreram com frequência muito maior que entre outros e é possível identificar relações preferenciais relativas à similaridade ou à complementaridade entre peças de conhecimento (Kay et. al, 2014).

Ainda que não se observe uma mudança geral da estrutura do conhecimento tecnológico, as relações mais importantes dentro da estrutura do conhecimento (a rede) podem se alterar, refletindo o surgimento de novas trajetórias tecnológicas. Para averiguar possíveis mudanças locais na estrutura do conhecimento tecnológico foi utilizada a medida de distância tecnológica por coocorrência para uma comparação nos três períodos (1988, 1998 e 2008) seguindo a metodologia de Hinze et al. (1997). Para isto, é determinada a frequência de coocorrência de cada campo técnico com todos os demais. Quanto 
maior a frequência de coocorrência entre dois códigos, maior a força da relação entre eles e menor a distância tecnológica. A distância entre campos tecnológicos é indicada pela proporção de coocorrência entre os códigos de classificação:

$$
d_{i j}=\frac{l_{i j}}{l_{i}}
$$

tal que $d_{i j}$ é a distância entre os campos tecnológicos $i$ e $j ; l_{i j}$ é a quantidade de links entre $i$ e $j$ e $l_{i}$ é o total de ocorrências de $i$ na base de patentes. Os dados de distância tecnológica formam uma matriz assimétrica, visto que, a importância relativa de $i$ para $j$ não é necessariamente igual à importância relativa de $j$ para $i$.

Uma forma de descrever e explorar as propriedades de uma rede consiste em estimar a correlação entre as medidas de centralidade para todos os nós. Se essas medidas têm correlação alta, seja negativa ou positiva, há um indicativo da forma como os nós se agrupam ou se distribuem na rede.

As medidas de centralidade de um nó ou vértice numa rede determinam a importância relativa de cada nó. A centralidade de grau se define como o número de arestas incidentes sobre um vértice e indica as relações diretas de um campo tecnológico. A centralidade de proximidade se define como uma proporção entre o número de vértices na rede e a distância de um vértice a todos os demais. Por isso, a proximidade é uma medida da posição global do campo tecnológico em relação à rede. No Gráfico 1(A), a centralidade de proximidade está positivamente relacionada com a centralidade de grau, o que indica que os nós mais centrais para rede também são os que possuem mais relações diretas.

A centralidade de intermediação mede quantas vezes um vértice é o caminho mais curto entre dois outros vértices escolhidos aleatoriamente. De maneira geral, a intermediação mede a importância de um nó localmente para mediar relações entre outros nós. O Gráfico 1(B) apresenta a evolução no tempo da relação entre a centralidade de grau e a centralidade de intermediação. Os pontos em roxo escuro representam os dados para 1988 e uma relação positiva entre o grau e a intermediação. A comparação dos três períodos, representados pelas diferentes tonalidades, mostra que houve crescimento do grau e redução da centralidade de intermediação. Em termos da estrutura da rede, isso quer dizer que à medida que a rede se tornou mais densa e os campos tecnológicos se tornaram muito mais inter-relacionados, os campos tecnológicos intermediários que deveriam fazer a ponte entre outros campos técnicos distintos perderam importância relativa porque a rede já estava bastante conectada.

\section{Gráfico 1. Evolução da Relação entre as Medidas de Centralidade}
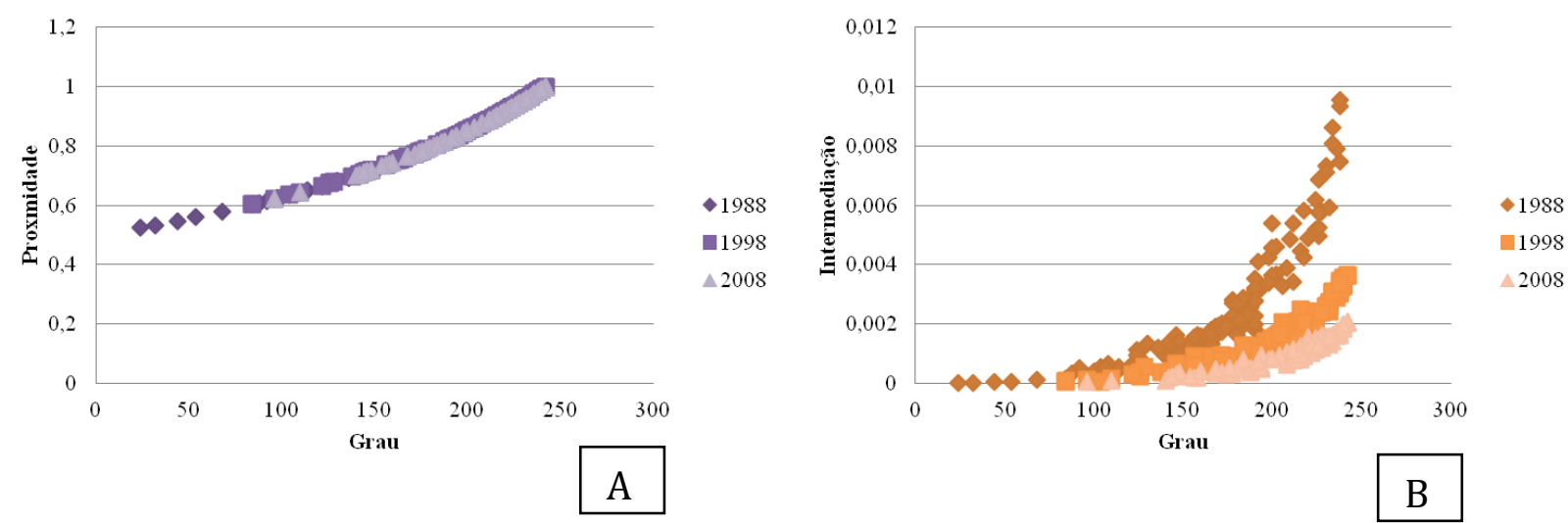

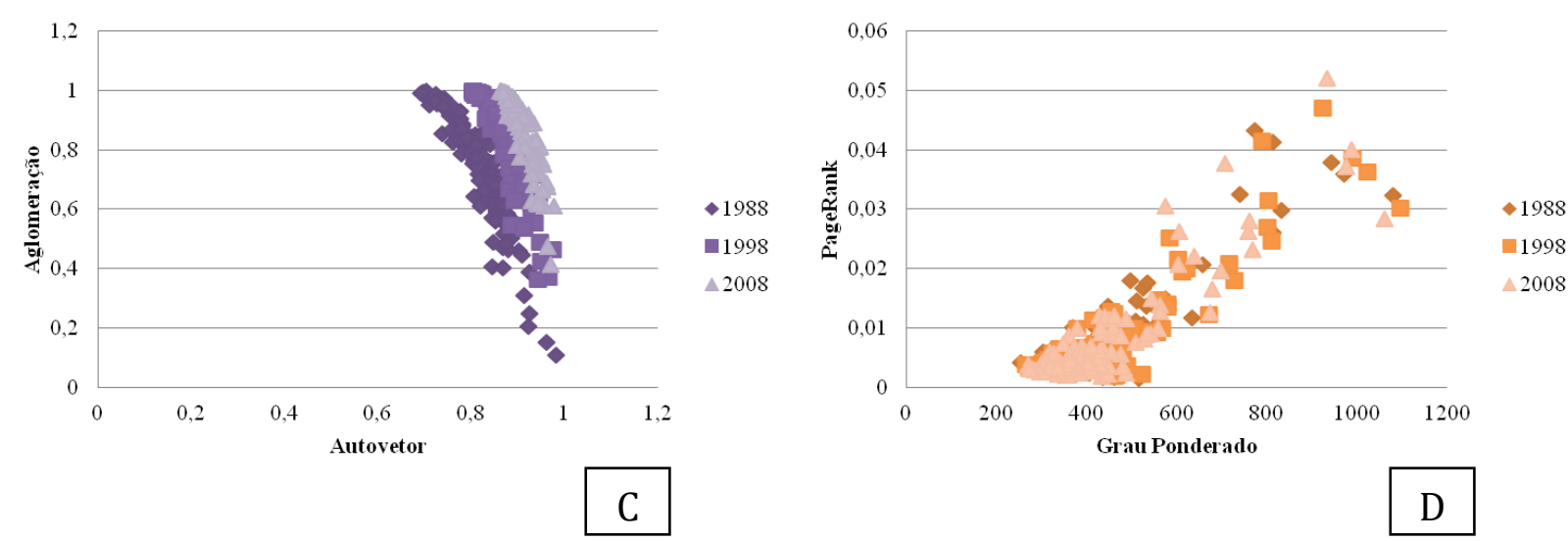

A centralidade de autovetor é uma medida de influência na rede que qualifica um vértice como central de acordo com a importância dos vértices com que ele se relaciona. Outra medida estrutural da rede, o coeficiente de agrupamento (clustering), mede o grau com que os nós tendem a formar grupos localmente. No Gráfico 1(C), as curvas negativamente inclinadas representam a evolução da relação entre a centralidade de autovetor e o coeficiente de agrupamento no tempo e as tonalidades indicam os dados de cada período. Os pontos em roxo mais escuro, que representam os dados de 1988, apontam que os campos tecnológicos formavam grupos bem conectados entre si e com relações externas mais fracas. Com o passar do tempo, o adensamento da rede diminuiu a distância entre os grupos, ou seja, está se formando um único grupo com campos tecnológicos bem conectados entre si.

O PageRank é uma medida derivada do conceito de influência de nós vizinhos. A medida consiste num grau ponderado pela força de cada relação, o qual evidencia mais as diferenças entre os nós do que o grau simples porque existem ligações que ocorrem com mais frequência na rede. Os nós que são relativamente mais importantes na rede possuem muitas ligações, mas principalmente, são as ligações mais fortes as que os diferenciam dos outros nós. No Gráfico 1(D), as tonalidades indicam a evolução da relação entre o grau ponderado e o PageRank. A maioria dos pontos está concentrada no canto inferior esquerdo do gráfico porque o grau médio ponderado não varia muito ao longo do tempo. Mas um pequeno conjunto de nós se manteve nos três períodos um pouco mais afastado do grupo maior. São os campos tecnológicos de maior centralidade porque possuem as relações mais fortes na rede. Estes campos tecnológicos são considerados hubs porque além de estarem conectados a muitos outros campos tecnológicos concentram as ligações consideradas mais fortes da rede. Os quatro campos tecnológicos que tiveram o maior grau ponderado entre os 121 analisados foram Processos ou aparelhos físicos ou químicos em geral (B01), Elementos elétricos básicos (H01), Medição e testes (G01) e Ciência médica ou veterinária; higiene (A61).

As redes bibliométricas e os scientograms são usados para visualizar as relações entre campos de conhecimento e identificar dentro da estrutura de conhecimento os principais tópicos de pesquisa; como esses tópicos se relacionam entre si e como determinados tópicos evoluíram no tempo. Recentemente, muitos estudos também têm proposto uma representação dos campos tecnológicos como um mapa a partir dos dados de patentes para comparações de portfólios e análises dinâmicas (Schoen et al., 2012; Kay et al., 2014; Leydesdorff, Kushnir, Rafols, 2014; Nakamura, Suzuki, Sakata, Kajikawa, 2015). Os métodos de análise utilizados nos scientograms são aplicados para a análise dos mapas de patentes e dos campos tecnológicos. Na análise de redes bibliométricas se utiliza uma combinação de técnicas de mapping e clustering. Neste trabalho, utilizou-se a técnica unificada de mapping e clustering proposta por Waltman e Noyons (2010). Os grafos foram gerados com auxílio do software Pajek, e a visualização dos mapas com o VOSviewer. 
O conhecimento tecnológico é cumulativo. Portanto, a estrutura de conhecimento refletida numa rede num período expressa o conjunto de inter-relações de todas as peças de conhecimento acumuladas até esse preciso momento no tempo. A Figura 1(A) representa a rede de conhecimento tecnológico acumulado até 2008 representada a partir do número de campos técnicos (IPCs) num nível de desagregação de 4-dígitos para o total de patentes depositadas na EPO até esse momento. O tamanho de cada campo tecnológico representa o grau ponderado ou a soma do peso de todos os links que cada um possui. A largura da aresta representa a força de uma relação, mas apenas as relações mais fortes aparecem na imagem. As cores representam as comunidades formadas a partir do método de identificação de comunidades VOS Clustering. A Figura 1(B) representa o mapa de calor da rede, sendo que a área do mapa com a maior concentração de ligações tem cor vermelha. Trata-se dos campos tecnológicos de maior centralidade de grau ponderado (B01, H01, G01 e A61).

O grau ponderado ou a importância relativa de um campo tecnológico não depende da propensão do mesmo para produzir patentes. Isto foi atestado com o coeficiente de correlação entre o grau ponderado e a frequência do campo tecnológico não significativo. A importância relativa de um campo tecnológico depende das características da própria tecnologia e daquelas com as quais possui ligações. Assim, os hubs identificados na imagem indicam campos tecnológicos que podem estar associados a tecnologias permeáveis porque fizeram a integração da base de conhecimento tecnológico formando relações fortes com vários campos tecnológicos distintos. Mesmo com o surgimento de novas ligações e com o adensamento da rede, os campos tecnológicos mais centrais permaneceram com essa posição relativa por concentrarem as relações mais fortes.

\section{Figura 1. Evolução da estrutura de conhecimento (1988-1998-2008)}

A

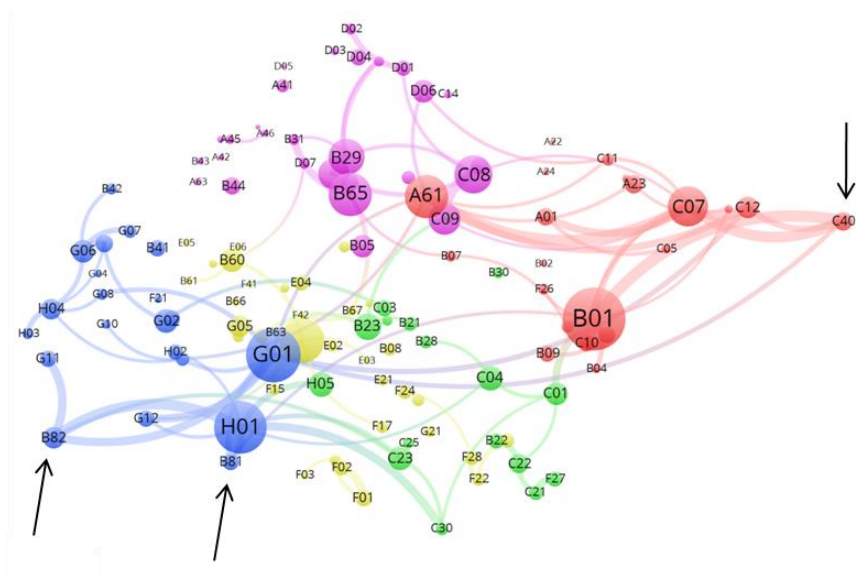

B

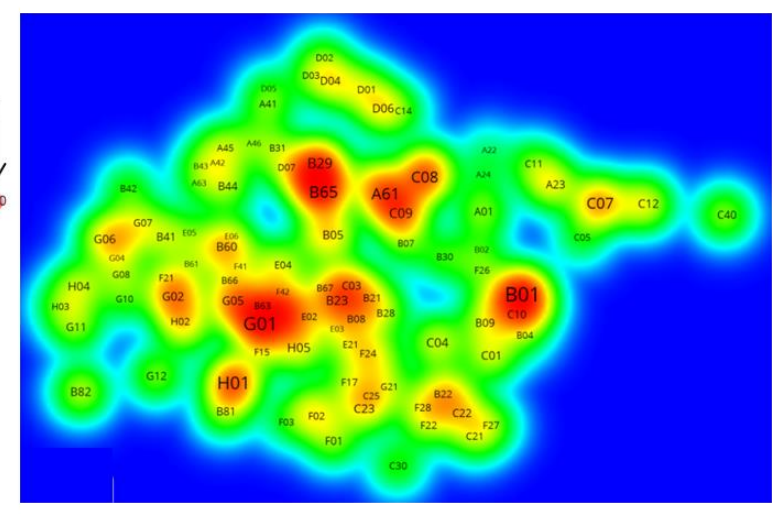



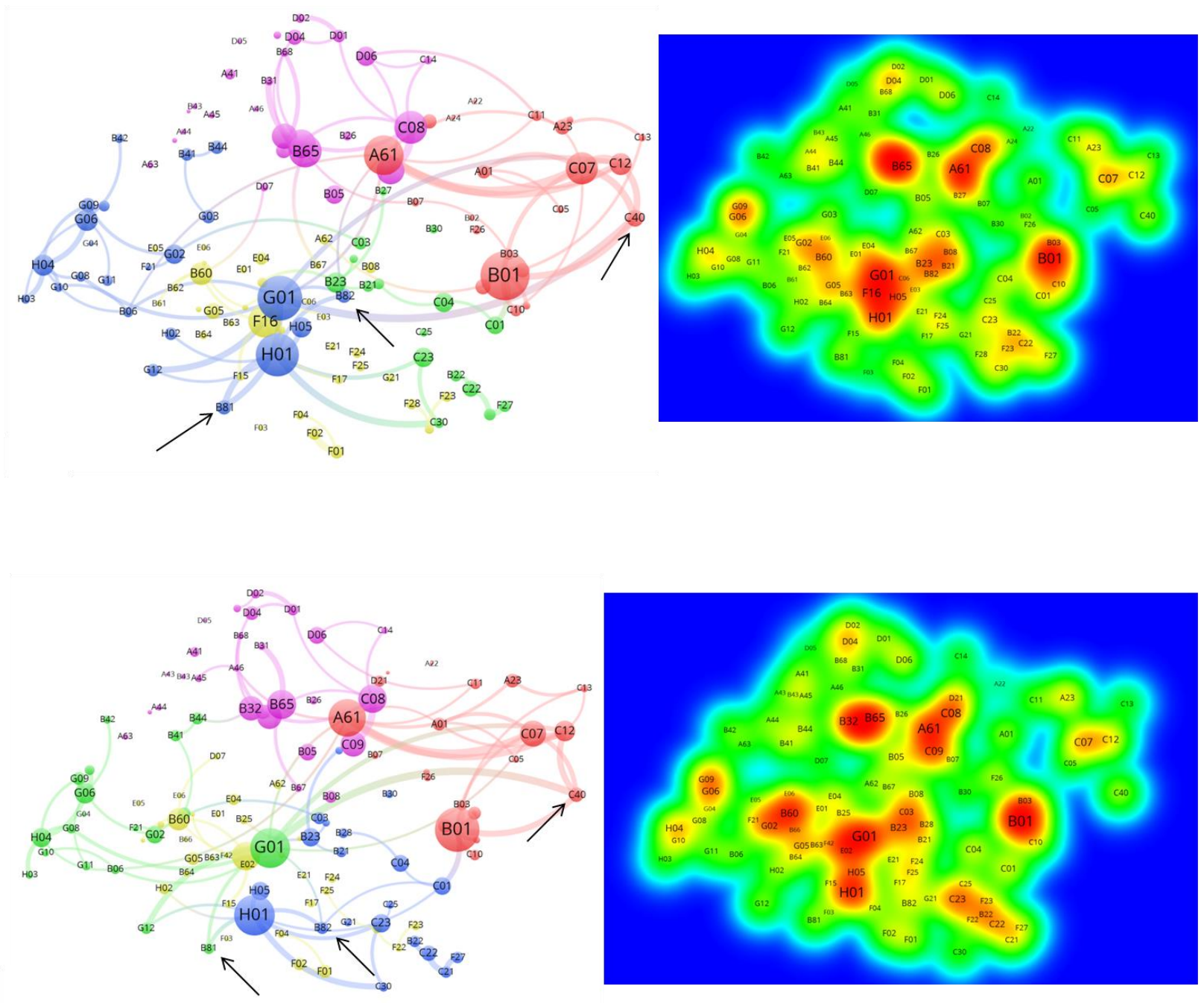

Figura 2. Principais relações - componente gigante (2008)

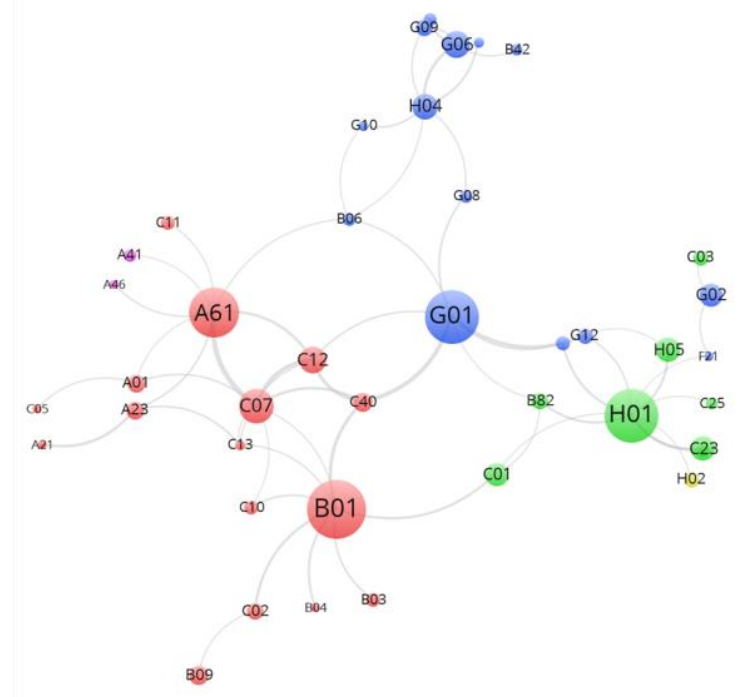




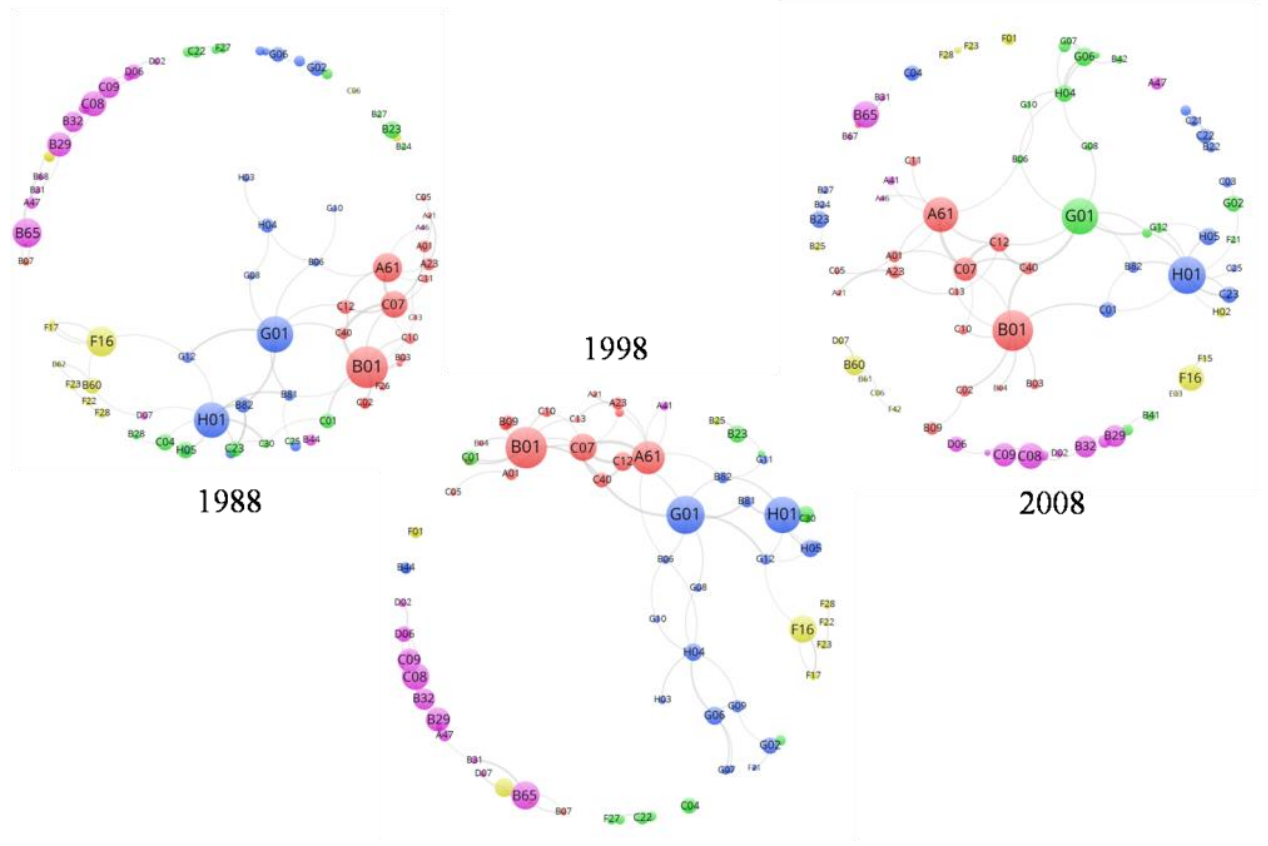

Do ponto de vista da estrutura do conhecimento tecnológico, os hubs também podem ser considerados um fator para a estabilidade a que se referiram outros trabalhos com análise de patentes (Hinze et al., 1997; Kay et al., 2014). O amadurecimento dos paradigmas tecnológicos está associado ao crescimento da complexidade da estrutura do conhecimento e, portanto, a uma perda de maleabilidade da mesma. Ao mesmo tempo, a cumulatividade do conhecimento e a irreversibilidade das trajetórias tecnológicas levam a que certos padrões bem definidos de combinação entre tecnologias se tornem comuns e sejam dificilmente superados por novos.

Mas, na rede também é possível observar relações fortes que procedem de campos tecnológicos que estão menos presentes na base de patentes. Essas relações, apesar de serem consideradas fortes, são mais propensas a mudar do que as outras. Os campos tecnológicos que ainda não estão amadurecidos podem ter um crescimento das suas ligações de forma mais diversificada ou ainda podem seguir uma relação preferencial a partir de uma nova combinação. Com isso, ao longo do tempo a distância tecnológica entre dois campos tecnológicos pode mudar. A força de um link revela a importância da sua interconexão com a rede. Quando uma relação ocorre entre dois campos tecnológicos que aparecem com uma frequência acima da média na base de patentes, a relação tende a registrar menos alterações ao longo do tempo. Mas, se a relação ocorre com ao menos um dos campos tecnológicos -sendo ainda pouco frequente na base-, com o passar do tempo o surgimento de novas relações alterará a importância dos seus links com mais facilidade.

A Tabela 2 mostra a evolução da distância tecnológica para os períodos de 1988, 1998 e 2008 . A distância tecnológica $d_{i j}$, como já foi visto, mostra a importância da relação entre os campos tecnológicos $i$ e $j$. Na tabela, as duas primeiras colunas são os códigos IPC's a 3 dígitos que representam os pares de campos tecnológicos e as colunas seguintes indicam a força da relação entre eles medida pela distância tecnológica $d_{i j}$ para cada período $(1988,1998$ e 2008). As duas últimas colunas são os valores referentes à variação da distância tecnológica de um período para o outro. A maior parte das relações sofre pequenas alterações no peso relativo, mas há casos importantes de mudanças que se observam para as relações dos campos tecnológicos B81 (Tecnologia das Microestruturas), B82 (Nanotecnologia) e C40 (Tecnologia Combinatória). 
Entre 1988 e 1998, o B81 (Tecnologia das Microestruturas) aumentou o peso relativo das suas ligações com H01 (Elementos elétricos básicos) e G01 (Medição e teste). Por outro lado, entre 1998 e 2008 as conexões deste campo com outros IPCs se tornou mais variada, o que reduziu a importância relativa das conexões com as tecnologias de núcleo (H01 e G01). Portanto, no desenvolvimento de B81 ocorreram mudanças que apontam para o surgimento de uma nova trajetória tecnológica, assim como um entrave à continuidade na direção que seguia anteriormente. $\mathrm{O}$ mesmo ocorreu com B82 (Nanotecnologia), cuja diversificação das relações ao longo do tempo levou à redução da importância das conexões mais fortes nos dois períodos analisados. No terceiro exemplo, o C40 (Tecnologia Combinatória) manteve as quatro ligações com peso relativo elevado entre 1988 e 1998, mas as ligações com C12 (Bioquímica) e G01(Medição e teste) se tornaram mais importantes relativamente.

Tabela 2 - Evolução do peso das relações entre 1988-2008 (IPC 3 dígitos)

\begin{tabular}{|c|c|c|c|c|c|c|c|c|c|c|c|c|}
\hline IPC i & IPC j & d 1988 & d 1998 & d 2008 & Var 1 Var 2 & IPC $i$ & IPC $\mathbf{j}$ & d 1988 & d 1998 & d 2008 & Var 1 & Var 2 \\
\hline $\mathrm{A} 01$ & $\mathrm{C} 07$ & 0,39 & 0,35 & 0,28 & $-0,12-0,19$ & $\mathrm{C} 05$ & $\mathrm{~A} 01$ & 0,27 & 0,24 & 0,24 & $-0,10$ & 0,00 \\
\hline A21 & 23 & & 0,44 & 0,45 & $0,14 \quad 0,01$ & & 1 & & & 0,50 & 0,21 & 0,10 \\
\hline A61 & $\mathrm{C} 07$ & 39 & 0,37 & 0,31 & $-0,06-0,16$ & $\mathrm{COS}$ & $\mathrm{C} 08$ & 44 & 15 & 0,42 & 0,03 & $-0,07$ \\
\hline B01 & $\mathrm{C} 07$ & 0,31 & 0,28 & 0,22 & $-0,10-0,21$ & $\mathrm{C}$ & B01 & 34 & 0,32 & 0,30 & $-0,04$ & $-0,06$ \\
\hline B03 & B01 & 0,25 & 0,29 & 0,31 & $0,15 \quad 0,06$ & $\mathrm{C} 12$ & $\mathrm{C} 07$ & 50 & 0,56 & 0,47 & 0,11 & $-0,16$ \\
\hline B04 & B01 & 0,33 & 0,35 & 0,36 & $0,04 \quad 0,02$ & $\mathrm{C} 12$ & A61 & 38 & 0,46 & 0,42 & 0,21 & $-0,09$ \\
\hline B06 & G01 & 0,28 & 0,29 & 0,25 & $0,01-0,11$ & $\mathrm{C} 12$ & G01 & 0,27 & 0,29 & 0,29 & 0,06 & 0,01 \\
\hline B06 & A61 & 0,24 & 0,22 & 0,23 & $-0,08 \quad 0,03$ & $\mathrm{C} 23$ & $\mathrm{H} 01$ & 0,29 & 0,33 & 0,32 & 0,15 & $-0,04$ \\
\hline B09 & $\mathrm{C} 02$ & 0,25 & 0,21 & 0,22 & $-0,15 \quad 0,04$ & $\mathrm{C} 3$ & $\mathrm{H} 01$ & 55 & 0,56 & 0,52 & 0,02 & $-0,07$ \\
\hline B31 & B65 & 0,50 & 0,51 & 0,50 & $0,01-0,01$ & $\mathrm{C} 4$ & B01 & 78 & 0,57 & 0,54 & $-0,27$ & $-0,05$ \\
\hline B32 & $\mathrm{C} 08$ & 0,29 & 0,29 & 0,26 & $0,02-0,10$ & $\mathrm{C} 4 \mathrm{C}$ & $\mathrm{C} 07$ & 73 & 0,61 & 0,44 & $-0,16$ & $-0,29$ \\
\hline B32 & B29 & 0,28 & 0,26 & 0,21 & $-0,10-0,17$ & $\mathrm{C} 4 \mathrm{C}$ & $\mathrm{C} 12$ & 42 & 0,57 & 0,55 & 0,33 & $-0,03$ \\
\hline B42 & G06 & 0,24 & 0,26 & 0,25 & $0,07-0,03$ & $\mathrm{C} 4 \mathrm{C}$ & G01 & 33 & 0,65 & 0,67 & 0,99 & 0,03 \\
\hline B62 & B60 & 0,23 & 0,26 & 0,28 & $0,09 \quad 0,08$ & D02 & D01 & 33 & 0,34 & 0,33 & 0,03 & $-0,02$ \\
\hline B67 & B65 & 0,30 & 0,29 & 0,29 & $-0,01-0,02$ & F0 & F02 & 0,38 & 0,39 & 0,35 & 0,04 & $-0,09$ \\
\hline B68 & A47 & 0,38 & 0,39 & 0,34 & $0,04-0,13$ & F1: & F16 & 0,27 & 0,29 & 0,29 & 0,08 & 0,01 \\
\hline B68 & B29 & 0,26 & 0,24 & 0,17 & $\begin{array}{lll}-0,09 & -0,29\end{array}$ & $\mathrm{~F} 22$ & F28 & 0,25 & 0,21 & 0,18 & $-0,15$ & $-0,14$ \\
\hline B81 & H01 & 0,46 & 0,56 & 0,37 & $0,21-0,34$ & $\mathrm{~F} 27$ & $\mathrm{C} 21$ & 0,30 & 0,33 & 0,33 & 0,09 & 0,00 \\
\hline B81 & G01 & 0,38 & 0,53 & 0,31 & $0,38-0,41$ & G0 & G06 & 0,31 & 0,36 & 0,36 & 0,15 & 0,01 \\
\hline B82 & H01 & 0,71 & 0,39 & 0,38 & $-0,46 \quad 0,00$ & G0 & G01 & 0,30 & 0,32 & 0,31 & 0,06 & $-0,03$ \\
\hline B82 & G11 & 0,57 & 0,21 & 0,10 & $-0,64-0,52$ & G0 & G06 & 0,24 & 0,28 & 0,27 & 0,16 & $-0,06$ \\
\hline $\mathrm{B}$ & G0 & 0,5 & 0,28 & 0,21 & $-0,50-0,27$ & G1 & G01 & 0,56 & 0,55 & 0,54 & $-0,02$ & $-0,02$ \\
\hline B82 & $\mathrm{C}_{2}$ & 0,29 & 0,05 & 0,06 & $-0,82 \quad 0,14$ & G12 & $\mathrm{H} 01$ & 0,25 & 0,25 & 0,26 & 0,00 & 0,05 \\
\hline $\mathrm{C} 0$ & B & 0,40 & 0,40 & 0,39 & $-0,01-0,01$ & H03 & H04 & 0,24 & 0,34 & 0,34 & 0,39 & 0,01 \\
\hline C02 & B01 & 0,41 & 0,43 & 0,43 & $0,03 \quad 0,00$ & H05 & $\mathrm{H} 01$ & 0,34 & 0,38 & 0,37 & 0,10 & $-0,01$ \\
\hline
\end{tabular}

Nota: IPC i e IPC j são os códigos IPC's a 3 dígitos; d1988 é a distância tecnológica entre IPC i e IPC j em 1988 ; d1988 é a distância tecnológica em 1998; d2008 é a distância tecnológica em 2008; Var 1 é a variação da distância tecnológica entre 1988 e 1998; Var 2 é a variação da distância tecnológica entre 1998 e 2008.

Fonte: Elaboração própria.

Os casos dos campos tecnológicos B81 (Tecnologia das Microestruturas), B82 (Nanotecnologia) e C40 (Tecnologia Combinatória) demonstram o que se propôs sobre a importância de observar a evolução da força das relações no tempo para identificar mudanças na estrutura da base de conhecimento. Os campos tecnológicos menos desenvolvidos, seja por serem mais recentes ou mesmo por serem pouco frequentes na base, estão mais propensos a apresentar mudanças na força de suas relações e, portanto, na distância tecnológica à medida que se tornam mais interrelacionados com outros campos tecnológicos diferentes.

Outra forma de observar a estabilidade da base de conhecimento é mediante a mobilidade dos agrupamentos formados pelos campos tecnológicos ao longo do tempo. A mobilidade de um campo 
tecnológico entre grupos dominantes (paradigmas tecnológicos) envolve uma transformação na oportunidade tecnológica (possibilidades de exploração de novos caminhos), pois revela como uns paradigmas afetam a outros no estabelecimento das trajetórias do progresso técnico. Já o crescimento da importância relativa de um campo tecnológico dentro de seu paradigma está associado à cumulatividade e a um menor grau de pervasividade. A Tabela 3 identifica oito micro-paradigmas tecnológicos os quais representam os clusters que foram formados pelo agrupamento de campos técnicos. A evolução da estrutura da base de conhecimento e dos micro-paradigmas ao longo de três períodos descreve a importância relativa dos campos tecnológicos que pode ser alta (A), média (M) ou baixa (B).

\section{Tabela 3. Número de IPCs a 3 dígitos associados a cada micro-paradigma de acordo com sua importância tecnológica por periodo.}

\begin{tabular}{|c|c|c|c|c|c|c|c|c|c|c|c|c|c|}
\hline & & \multicolumn{4}{|c|}{1988} & \multicolumn{4}{|c|}{1998} & \multicolumn{4}{|c|}{2008} \\
\hline & & B & $\mathbf{M}$ & $\mathbf{A}$ & Total & $\mathbf{B}$ & $\mathbf{M}$ & $\mathbf{A}$ & Total & $\mathbf{B}$ & M & $\mathbf{A}$ & Total \\
\hline I. & Análise, medidas e controle; Máquinas elétricas e Tecnologia Audio-visual & 19 & 1 & 2 & 22 & 17 & 4 & 2 & 23 & 19 & 4 & 2 & 25 \\
\hline II. & Engenharia Química; Farmacêutica, Cosmética; Agricultura e Alimentos & 17 & 1 & 2 & 20 & 17 & 2 & 2 & 21 & 16 & 2 & 2 & 20 \\
\hline III. & Elementos mecânicos, Engenharia civil e Transporte & 16 & 1 & 1 & 18 & 16 & 2 & & 18 & 14 & 2 & & 16 \\
\hline IV. & Máquinas e Motores; Aparatos e processos com troca de calor & 11 & & & 11 & 11 & & & 11 & 11 & & & 11 \\
\hline V. & Máquinas-ferramentas; Materiais, metalurgia; Tecnologia de superfície & 13 & 4 & & 17 & 13 & 3 & & 16 & 14 & 3 & & 17 \\
\hline VI. & Manuseio e impressão, Processamento de materiais, Bens de consumo e & 9 & & 1 & 10 & 9 & 1 & & 10 & 9 & 2 & & 11 \\
\hline VII. & Bens de consumo e equipamentos; Processamento de materiais, textêis, papel & 13 & 4 & & 17 & 14 & 5 & & 19 & 14 & 4 & & 18 \\
\hline VIII. & Tecnologias espaciais e Armamento & 3 & & & 3 & 3 & & & 3 & 3 & & & 3 \\
\hline
\end{tabular}

Nota: Importância tecnológica Alta (A), Media (M), Baixa (B).

Elaboração própria.

O micro-paradigma de tecnologias de Análise, medidas e controle; Máquinas elétricas e Tecnologia Audio-visual é o mais variado [maior quantidade de campos tecnológicos diferentes]. Dois destes campos possuem alta importância relativa e não mudam de paradigma ao longo do tempo (persistência no núcleo). Porém, há entrada de outros campos tecnológicos no grupo de menor importância relativa. No micro-paradigma de Engenharia Química; Farmacêutica, Cosmética; Agricultura e Alimentos também há persistência no núcleo ao longo do tempo e não há mobilidade considerável de outros campos tecnológicos para dentro, o qual indica que o micro-paradigma ofereceu menos oportunidades relativas para campos tecnológicos externos. Nos micro-paradigmas de Elementos mecânicos, Engenharia civil e Transporte e de Manuseio e impressão, Processamento de materiais, bens de consumo e equipamentos houve mobilidade no núcleo que perdeu importância relativa. Os micro-paradigmas Máquinas e Motores; Aparatos e processos com troca de calor e também em Tecnologias espaciais e Armamento não sofreram mudanças, indicando pouca pervasividade e pouca oportunidade tecnológica para campos tecnológicos externos ao grupo. No micro-paradigma de Máquinas-ferramentas; Materiais, metalurgia; Tecnologia de superfície houve mudanças por mobilidade de campos técnicos; entre 1988 e 1998 houve a saída de um e entre 1998 e 2008 houve entrada de outro campo técnico diferente. No micro-paradigma Bens de consumo e equipamentos; Processamento de materiais, textêis, papel houve mobilidade de tecnologias no primeiro e no segundo período que foi acompanhada de redução do grau de pervasividade de um dos campos técnicos.

A existência de núcleos bem definidos na estrutura da base de conhecimento está associada a tecnologias que são mais pervasivas e a campos tecnológicos que tendem a estabelecer relações mais diversas e com força relativa maior, tornando a base mais complexa como um todo. 


\section{Conclusões}

Nas últimas décadas houve uma forte expansão da quantidade de patentes que trouxe consigo um aumento da quantidade de campos tecnológicos e de novas relações entre eles. No entanto, vários estudos apontam para a estabilidade da estrutura do conhecimento tecnológico. Com o surgimento de novos micro-paradigmas, a incerteza aumenta devido às muitas direções que a mudança tecnológica pode seguir. No entanto, à medida que amadurecem, a seleção tecnológica determina caminhos ou trajetórias que paulatinamente vão retirando flexibilidade da estrutura do conhecimento tecnológico. Neste sentido, este trabalho se propôs a analisar a evolução da estrutura do conhecimento tecnológico, concretamente no que se refere à complexidade e à estabilidade, associada ao amadurecimento dos paradigmas tecnológicos ao longo de três décadas. A base de conhecimento foi construída utilizando as classificações IPC a 3 dígitos associadas às patentes depositadas entre 1988 e 2008 na European Patent Office e a análise utilizou a metodologia aplicada a redes.

A estabilidade é uma característica relevante da rede de conhecimento tecnológico como um todo, mesmo quando analisada para um período longo de tempo. A rede possui relações fortes entre campos tecnológicos que se mantém ao longo do período analisado. Os núcleos que permaneceram fortes na estrutura de conhecimento tecnológico deram um certo grau de estabilidade ao longo do tempo. Mas, paralelamente surgiram também novas relações que mudaram a distância relativa entre campos tecnológicos. A classificação das relações quanto a sua força permitiu visualizar mudanças na posição de alguns campos tecnológicos na estrutura da rede e que podem indicar novas oportunidades de desenvolvimento tecnológico pela recombinação de peças de conhecimento. Ainda que os campos tecnológicos que formam os núcleos da rede sejam os mesmos, a estrutura do conhecimento tecnológico muda sempre que surgem novas oportunidades tecnológicas. Essas mudanças são observadas na forma como os campos tecnológicos se reagrupam. Pelo efeito da cumulatividade do conhecimento, os núcleos tendem a permanecer constantes ao longo do tempo. Mas, a oportunidade tecnológica pode levar a mudanças na importância relativa desses núcleos, nas trajetórias dos campos tecnológicos que dependem do núcleo e ao surgimento de novos núcleos.

O mapa de patentes ao permitir observar a estrutura de conhecimento tecnológico como uma rede resulta em implicações importantes para a questão da diversificação tecnológica, pois a especialização em uma tecnologia envolve uma possível evolução para as outras tecnologias com que ela se relaciona. Assim, a especialização em uma tecnologia pode implicar que seja tão ou mais importante integrar peças de conhecimento tecnológico de campos técnicos distintos do que se especializar em um campo técnico. Além disso, bases de conhecimento com peças pouco relacionadas na rede podem representar bases de conhecimento ainda pouco maduras e a oportunidade tecnológica pode vir pela integração, ainda sub explorada, com outros campos técnicos. Dessa forma, a estrutura de rede do conhecimento tecnológico coloca em evidência a importância da diversificação através de novas relações entre campos técnicos e pode revelar áreas tecnológicas pouco exploradas que são centrais para o desenvolvimento de outras tecnologias. Desde esse ponto de vista, os mapas de patentes podem auxiliar nas decisões de políticas públicas para o desenvolvimento de sistemas de inovação em países da periferia pela capacidade de indicar o desenvolvimento tecnológico no tempo e revelar as áreas de potencial para interrelações entre tecnologias ainda pouco exploradas em comparação com os países centrais. 


\section{Referências Bibliográficas}

Aharonson, B. S., Schilling, M. A. (2016). Mapping the technological landscape: Measuring technology distance, technological footprints, and technology evolution. Research Policy, 45(1), 81-96.

Benson, C. L., \& Magee, C. L. (2013). A hybrid keyword and patent class methodology for selecting relevant sets of patents for a technological field. Scientometrics, 96, 69-82.

Benson, C. L., \& Magee, C. L. (2015). Technology structural implications from the extension of a patent search method. Scientometrics, 102, 1965-1985.

Boschma, R., Balland, P.-A., \& Kogler, D. F. (2015). Relatedness and technological change in cities: The rise and fall of technological knowledge in US metropolitan areas from 1981 to 2010. Industrial and Corporate Change, 24, 223-250.

Breschi, S., Lissoni, F., Malerba, F. (2003). Knowledge-relatedness in firm technological diversification. Research Policy, 32(1), 69-87.

Dosi, G. (1984). Technical change and industrial transformation: the theory and an application to the semiconductor industry. Springer.

Fleming, L. (2001). Recombinant uncertainty in technological search. Management Science, 47(1), 117132.

Fontana, R., Nuvolari, A., \& Verspagen, B. (2009). Mapping technological trajectories as patent citation networks. An application to data communication standards. Economics of Innovation and New Technology, 18(4), 311-336.

Granstrand, O., Sjölander, S. (1990). The acquisition of technology and small firms by large firms. Journal of Economic Behavior \& Organization, 13(3), 367-386.

Granstrand, O., P. Patel and K. Pavitt (1997), "Multi-Technology Corporations: Why They Have 'Distributed' Rather than 'Distinctive Core' Competencies", California Management Review, 39, 8-25.

Hidalgo, C.A., Klinger, B., Barabasi, A.-L. \& Hausmann, R. (2007). The Product Space Conditions the Development of Nations. Science 317 (5837), 482-487.

Hinze, S., Reiß, T., Schmoch, U. (1997). Statistical analysis on the distance between fields of technology. Fraunhofer-Inst. Systems and Innovation Research.

Joo, S., \& Kim, Y. (2010). Measuring relatedness between technological fields. Scientometrics, 83, 435454.

Kajikawa, Y., Yoshikawa, J., Takeda, Y., \& Matsushima, K. (2008). Tracking emerging technologies in energy research: Toward a roadmap for sustainable energy. Technological Forecasting \& Social Change, $75,771-782$.

Kay, L., Newman, N., Youtie, J., Porter, A. L., Rafols, I. (2014). Patent overlay mapping: Visualizing technological distance. Journal of the Association for Information Science and Technology, 65(12), 24322443.

Levinthal, D.A. \& March, J.G. (1993). The Myopia of Learning. Strategic Management Journal, 14 (S2), 95-112. doi: 10.1002/smj.4250141009 
Leydesdorff, L., Kushnir, D., Rafols, I. (2014). Interactive overlay maps for US patent (USPTO) data based on International Patent Classification (IPC). Scientometrics, v. 98, n. 3, p. 1583-1599.

Malerba, F., Orsenigo, L. (1996). Schumpeterian patterns of innovation are technology-specific.Research policy, 25(3), 451-478.

Mina, A., Ramlogan, R., Tampubolon, G., Metcalfe, J. S. (2007). Mapping evolutionary trajectories: Applications to the growth and transformation of medical knowledge. Research Policy 36, 789-806.

Nakamura, H., Suzuki, S., Sakata, I., \& Kajikawa, Y. (2015). Knowledge combination modeling: The measurement of knowledge similarity between different technological domains. Technological Forecasting and Social Change, 94, 187-201.

Nooteboom, B. (2000). Learning and Innovation in Organizations and Economies. Oxford: Oxford University Press.

Ogawa, T., \& Kajikawa, Y. (2015). Assessing the industrial opportunity of academic research with patent relatedness: A case study on polymer electrolyte fuel cells. Technological Forecasting \& Social. Change, 90, 469-475.

Patel, P., K. Pavitt (1997), "The Technological Competencies of the World's Largest Firms: Complex and Path-Dependent, but not much Variety", Research Policy, 26, 141-156.

Rigby, D. L. (2015). Technological relatedness and knowledge space: Entry and exit of US cities from patent classes. Regional Studies, 49, 1922-1937.

Sahal, D. (1985). Technological guideposts and innovation avenues. Research policy, 14(2), 61-82.

Schoen, A., Villard, L., Laurens, P., Cointet, J. P., Heimeriks, G., Alkemade, F. (2012). The network structure of technological developments; technological distance as a walk on the technology map. In Science \& Technology Indicators (STI) Conference.

Teece, D. J., Rumelt, R., Dosi, G., \& Winter, S. (1994). Understanding corporate coherence: Theory and evidence. Journal of Economic Behavior \& Organization, 23, 1-30.

Veefkind, V., Hurtado-Albir, J., Angelucci, S., Karachalios, K., \& Thumm, N. (2012). A new EPO classification scheme for climate change mitigation technologies. World Patent Information, 34(2), 106111.

Verspagen, B. (2007). Mapping technological trajectories as patent citation networks: A study on the history of fuel cell research. Advances in Complex Systems, 10(01), 93-115. 